\title{
Combination of ECG and PPG Signals for Healthcare Applications: A Survey
}

\author{
Mahmoud M. Bassiouni ${ }^{1 *}$, Islam Hegazy ${ }^{2}$, Nouhad Rizk ${ }^{3}$, Sayed A. El-Dahshan ${ }^{1,4}$, Abdelbadeeh M. Salem ${ }^{2}$ \\ ${ }^{1}$ Department of Computer Science, Faculty of Computer and Information Technology, Egyptian E-Learning University \\ (EELU), 33 El-messah Street, Eldoki 11261, El-Giza, Egypt \\ ${ }^{2}$ Faculty of Computer and Information Science, Ain Shams University, Abbassia 11566, Cairo, Egypt \\ ${ }^{3}$ Computer Science Department, Director of Undergraduate Studies, Houston University, Texas 77204, USA \\ ${ }^{4}$ Department of Physics, Faculty of Science, Ain Shams University, Cairo 11566, Egypt
}

Corresponding Author Email: mbassiouni@eelu.edu.eg

https://doi.org/10.18280/ama_b.641-409

Received: 10 July 2021

Accepted: 5 November 2021

\section{Keywords:}

healthcare, biomedical signal processing, electrocardiogram (ECG), heart rate variability $(H R V)$, photoplethysmogram (PPG), health care systems, applications

\begin{abstract}
Most health care systems use various physiological signals to provide an accurate diagnosis performance. The main common signals functional in health care applications are the electrocardiogram (ECG) and photoplethysmogram (PPG). ECG signal represents the electrical cardiac activity of the heart, while the PPG signal measures the changes in the blood volume. There are several applications in which the ECG combined with PPG can be used in the field of medical health care. This survey illustrates the various applications that combine features from the ECG and PPG signals. The review manifests the techniques, methodologies used in the data acquisition, pre-processing of the signals. The feature extraction and classification phases for both ECG and PPG are explained. The limitations, challenges, and future directions for the combined application of ECG and PPG are clarified to solve the medical problems that existed, presented, and feasible. This study aims to increase the interest in applying the combination between ECG and PPG signals in more applications and to obtain optimal measurements related to cardiac activity.
\end{abstract}

\section{INTRODUCTION}

Health Care Systems are defined as systems for the enhancement of human health through monitoring various organs in the human body [1]. These systems improve human health through diagnosis, prevention, and cure of specific diseases, recovery, and treatment of physical and mental losses in the people [2]. Two of the main common heart signals used in the health care systems are the electrocardiogram (ECG), photoplethysmogram (PPG). On one hand, ECG is one of the most salient attributes for continuous monitoring of health. One cardiac cycle is composed of five main peaks known as $\mathrm{P}$, $\mathrm{Q}, \mathrm{R}, \mathrm{S}$, and T [3]. The obstacles of the ECG are related to the heart location, size, fat or thin body because if the body is fat, it will affect the readings, users' anatomy, and the electrode location concerning the structural location of the heart it can produce inaccurate or abnormal heart rate (HR) readings [4]. Therefore, to overcome these obstacles, ECG technology depends on the electrical impulses of the heart instead of the blood volume.

On the other hand, PPG is defined as an optimal methodology for the measurement of the changes in the blood volume at the surface of the skin. It is considered to be the nearest alternative to the ECG signal as it holds essential cardiovascular information $[5,6]$. All these merits of the PPG suffer from the inaccurate estimation of the HR and many other limitations such as the skin tone, different skin types, motion noises, and signal crossovers among others [7]. It is necessary to combine the capabilities of the ECG and the PPG signals to get the overall benefits of the features obtained from both signals. Figure 1 illustrates the ECG and PCG signals. Figure 1(a) shows the ECG signals in terms of waveforms, and the black circle represents the main ECG peaks while Figure 1(b) shows the PPG signal and its waveforms, and the black circles in subfigure (b) show the systole and diastole peaks.
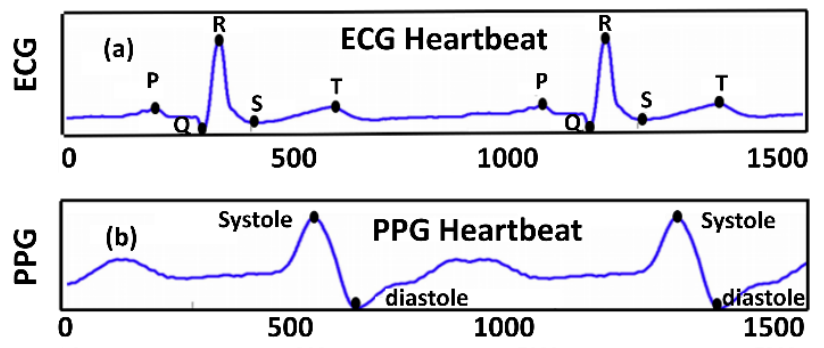

Figure 1. The ECG and its corresponding PPG signal

Various studies have shown that several features obtained from the PPG and ECG signals and mutual information between the PPG and ECG are necessary. In this study, the applications in which the ECG and PPG signals combined will be illustrated. One of the applications in which the PPG and ECG signal can be applied is hypertension [8]. Hypertension is a group of disorders in the blood vessels and the heart. It sometimes has some symptoms such as headache, chest pain, difficulty in the breath, but most of the people that have hypertension do not have any symptoms at all, therefore, it is sometimes called a "silent killer" $[9,10]$. Another application in which the ECG and PPG must be combined to have a better 
diagnosis is epilepsy [11]. This disorder affects almost $1 \%$ of the population. The treatment for this disease is based on antiepileptic drugs as they provide adequate treatment for about $70 \%$ of the patients with epilepsy $[12,13]$. Another application is known as driving under the influence (DUI) while intoxicated [14]. People that drive a motor vehicle while drinking alcohol are incapable of operating the motor vehicle safely. One of the main important applications that require the usage of the ECG and PPG is the continuous cufflessBP (Blood pressure). The damaging effects, discreteness, and the absence of an invasive method for the measurement of the continuous cuffless BP can lead to various heart problems [15, 16]. Finally, one of the most important applications of the ECG combined with the PCG signals is the estimation of respiratory sinus arrhythmia (RSA) [17]. The RR intervals are defined as the difference between two successive ECG waves. The RR intervals with different amplitudes are applied to allow the estimation of the respiratory rate and the temporal pattern of respiration. RSA is reflected in high-frequency HR oscillation which relates to cyclical changes in the HR that are equivalent to the respiratory cycle [18]. The main contributions of the survey are defined as follows:

- Investigation of the most important cardiovascular diseases in which the ECG and PPG were employed together to detect the existence of such diseases.

- Illustration of the methods and techniques used in the diagnosis of heart diseases based on the combination of ECG and PCG signals.

The rest of the paper is organized as follows. Section 2 presents the related work, while section 3 explains the methodology used based on the combination between ECG and PPG. Section 4 presents the discussion, and finally, section 5 manifests the conclusions and future work.

\section{LITERATURE REVIEW}

A related work of the previous studies using both ECG and PPG is defined in detail in terms of data capturing, filtering the signals, extracting features from it, and final diagnosis based on the performance results. Liang et al. [18] worked on subjects (S) captured from the MIMIC database. Each recording holds some physiological signals such as arterial blood pressure (ABP), ECG, and PPG. The number of subjects obtained was 121 participants. Pulse Arrival Time (PAT) is extracted from the R-wave of ECG signals and the foot of the PPG waveform. PPG features are obtained beat by beat. From these features, only 10 PPG features and the PAT features were used combined to classify blood pressure (BP) categories The classification was based on Bagged trees (BT), linear regression (LR), and K-nearest-neighbor (KNN), and $\mathrm{KNN}$ showed the highest F1-measure.

Lan et al. [19] worked on 43 subjects, 24 subjects have hypertension. The subjects were divided into 30 for training and 13 subjects for test. Six main features were obtained based on heart rate variability (HRV). Three of them are in the time domain known as the standard deviation of normal-to-normal intervals (SDNN), mean of the RR interval (MeanRR), square root of the mean square of all $\mathrm{NN}$, or the $\mathrm{RR}$ interval during a specific period (RMSSD). The other three are in the frequency domain and they involve normalized low-frequency power of HRV (LFnu), normalized high-frequency power of HRV (HFnu), low to high-frequency power ratio (LF/HF). The prediction methodology is based on multiple instance learning (MIL).

Ghosh et al. [20] estimated the continuous BP based on pulse transit time (PTT) that is estimated from ECG and PPG signals. A calibration process is constructed to form a calibration curve from the PTT to BP. In the experimental results, it was shown that 1 over PTT is more linearly related to the BP than the PTT. The root mean square error (RMSE) as a performance was applied using sparsity and without sparsity, it was shown that with sparsity methodology achieved the lowest RMSE. Ding et al. [21] applied a methodology to estimate the systolic blood pressure (SBP), diastolic blood pressure (DBP), and mean blood pressure (MBP) using 27 subjects with continuous BP as a reference. The PTT and pulse intensity ratio (PIR) were obtained from both the ECG and PPG signals.

Kachuee et al. [22] applied a set of stages for the estimation of the BP, a calibration method is applied to enhance the performance. The pre-processing is based on discrete wavelet transform (DWT), zeroing, and wavelet de-noising based on soft rigrsure-thresholding. The features obtained are physiological parameters which are PAT, HR, augmentation index, large artery Stiffness index, and inflection point area ratio, while the whole-based features are based on timedomain features obtain from ECG and PPG signals. Six different classifiers are applied which are LR, ADT, RFR, DT, and SVM. The results are obtained in the form of MAE and standard deviation. AdaBoost and RF showed the highest performance using the two types of feature extraction proposed over all other classifiers.

Sharifi et al. [23] proposed a methodology to classify three types of BP which are the SBP, DBP, and MBP. The data was captured from the MIMIC II dataset, and VMD was applied for de-noising. PTT and pulse intensity ratio (PIR) were extracted. The regression model is based on the multi-adaptive regression spline (MARS), and the performance measurements are based on the mean absolute difference (MAD), and percent root means squared difference (PRD). Tanveer and Hasan [24] applied a methodology for estimating BP. The data was obtained from a MIMIC I database, and about 39 patients were selected. A preprocessing methodology was applied to remove the baseline wandering and noise, Tunable-Q wavelet transform (TQWT) was used. After the signals are processed a non-uniform waveform segmentation is performed based on the extraction of the three successive ECG and PPG waveforms. The ECG and PPG waveforms are concatenated after normalization forming a feature vector of 513 samples. An artificial neural network long-short term memory (ANN-LSTM) network is proposed for the features obtained from the non-uniform segmentation. The results were expressed in terms of mean absolute error (MAE) and RMSE. Liu et al. [25] proposed a method for the prediction of BP using ECG and PPG signals. The data was collected and gathered from 35 five clinically stable patients. Fifteen of these patients have ventricular arrhythmias, while the remaining 20 have supraventricular arrhythmia. The poorquality PPG signals were improved using the Butterworthband pass filter and adaptive thresholding setting. Fifteen features were extracted based on the ECG, PPG signals, and the combination between them. The regression methods used are based on the decision trees (DT), support vector regression (SVR), Adaboost, and Random forest (RF). RF had the highest regression performance. 
$\mathrm{Xu}$ et al. [26] estimated the BP based on features obtained from ECG and PPG signals. The signals were collected from 7 volunteers. The pre-processing of the signals was based on a one-band pass finite impulse response (FIR) filter and an infinite impulse response (IIR) filter. The features were based on extracting the $\mathrm{R}$ peak, performing deviation on the PPG signal to obtain the maximum slope in the rising edge, calculating the PTT.

Then, fifteen parameters were obtained and they acted as an input to the backpropagation neural network (BNN). Yao et al. [27] implemented a methodology for the estimation of BP. The data were collected from the MIMIC II database, 2000 signals were collected. The ECG and PPG signals were filtered using wavelet analysis and before reconstruction, a low-pass filter was applied to coefficients. The features that were obtained from this study are about 70 and they were ranked based on the correlation from the greatest to the least. The classification was based on DT, AdaBoost, LR, random trees (RT), support vector machine (SVM), and ridge regression. AdaBoost classifier showed the highest accuracy performance over the other classifiers.

Jan et al. [28] proposed a method for determining the efficiency of the HRV and pulse rate variability (PRV) in estimating five types of controlled breathing such as natural placed breathing (NPB), rapid deep breathing (RDB), slow and shallowing breathing (SSB), rapid and shallow breathing (RSB). The number of subjects used in this study is 30 subjects classified as young and seniors, and the HRV and PRV were obtained. On one hand, the results showed that in the young group the Ratio $_{\mathrm{RR}}$ based on HRV was higher than that of the $\mathrm{PRV}$ in RDB, SDB, RSB, and SSB, but the Ratio ${ }_{\mathrm{RR}}$ of the PRV is higher than that of the HRV in NBP and the rest cases. Ratio $_{\mathrm{RR}}$ is the ratio of the range of percentage recurrence obtained by self-recurrence. In other words, Ratio ${ }_{\mathrm{RR}}$ indicates the coupling of the two signals if its value is nearly equal to one. On the other hand, the results showed that in the senior group the Ratio ${ }_{\mathrm{RR}}$ based on HRV was higher than that of the PRV in RDB, SDB, RSB SSB, and NBP in motion and rest cases.
Wang et al. [29] proposed a methodology to identify people that consumed alcohol. The methodology is based on capturing data based on a specific data acquisition system. In the pre-processing stage, the signals are analyzed using fast Fourier transform (FFT) to determine the energy and its distribution. A Kaiser window-based digital FIR filter is applied to filter noise and retain the original parts of the ECG and PPG signals. The segmentation is based on the modulesmaxima wavelet analysis, and this method ends with the detection of PPG signals and $\mathrm{R}$ detection. The features obtained are based on the combination of the ECG and PPG signals time intervals, QRS interval, and the mean of the intervals. Finally, the features are combined and sent forward to the support vector machine (SVM) for classification.

Vandecasteele et al. [30] worked on a methodology for the detection of an epileptic seizure. The data was captured from 11 patients with recordings of refractory epilepsy. The methodology is based on obtaining and capturing the HRV and PRV pulses, and the detection of the seizure. The ECG signal is filtered using a notch filter to remove the powerline interference, while the PRV is obtained for the detection of the seizure. Then, the HRV and PRV act as input, and a classification method is applied for extracting the most efficient features. The features are the HR peak, mean of the HR base, a standard deviation of the HR base multiplied by HR peak.

\section{COMPUTATIONAL INTELLIGENCE PARADIGMS FOR THE COMBINATION OF ECG AND PPG SIGNALS}

This section represents the main phases of various healthcare systems based on ECG and PPG Signals. The phases are data acquisition, pre-processing, features extraction, classification, and regression. Figure 2 shows the common techniques used in the former phases for ECG and PPG healthcare systems.

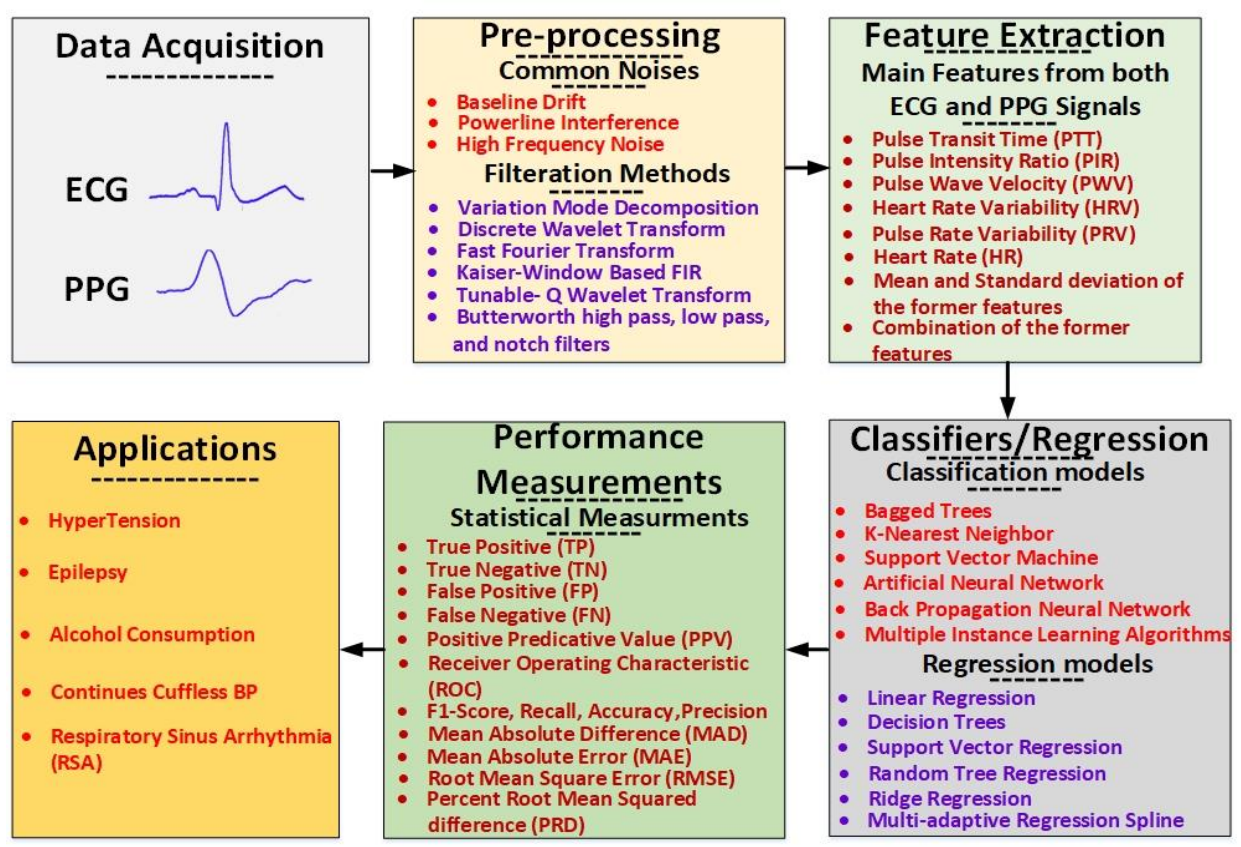

Figure 2. Generic architecture for the main stages and the common techniques applied using the combination of ECG and PPG signals 


\subsection{Data acquisition}

In this phase, the ECG and PPG signals are collected for further processing. In the applications in which the ECG and PPG signals are applied, some studies used data from an online source containing the ECG and PPG signals of the individuals, while other studies gathered and collected the signals from a subject using specific devices. In the estimation of hypertension in the individuals, Liang et al. [18] used data obtained from an open dataset that was used in most of the hypertension studies which is known as the Medical Information Mart for Intensive Care (MIMIC) database [31]. The (MIMIC) I database was used by Tanveer and Hasan [24] for the analysis of cuffless BP.

Moreover, (MIMIC) II database was used in different studies [22, 23, 27] for estimating different types of systole and diastole blood pressure, while some studies [20] used other devices to capture data such as BioRadio.This device is a biomedical device with channels to record and transmit a fusion of human physiological signals. Ding et al. [21] used one or two-lead electrodes placed on the arms to obtain the ECG signals and a PPG sensor on the left index finger to capture PPG signals. Liu et al. [25] used a multiparameter monitoring system known as BeneVision N12 to acquire synchronous ECG and PPG signals for patients in supine positions.

In the estimation of the variances of RSA [24], the ECG and PPG signals are captured from a polysomnography system with an adapted sampling frequency and an A/D converter, and for the identification of the alcohol consumption. Xu et al. [26] used a PowerLab 8/30 device to record the ECG and PPG signals. Finally, in the detection of epileptic seizures, some wearable devices were used to capture ECG such as the Faros device and it was configured in one channel representation. In other words, only a single lead was used to gather the ECG. Another device was used to capture the PPG signal known as the Empactica E4 smartwatch that is a wrist-worn PPG device. These are considered the most commonly used devices, systems, and methodologies for capturing the ECG and PPG signals combined for a specific purpose.

\subsection{Preprocessing}

In this phase, the ECG and the PPG signals must be filtered and de-noised for further extraction of the features from both signals. For some purposes and applications, the ECG and PPG signals were used preprocessed, filtered, and ready for feature extraction using a filtered methodology applied on both signals $[20,25]$. Others provided a pre-processing mechanism without specifying its details or main components $[18,19]$. In some studies, a specific filtering methodology was applied for each of the ECG and PPG signals separately. For instance, a preprocessing methodology was proposed by Sharifi et al. [23] based on decomposing each signal to 8 modes using VMD [32], and if there is a difference in the sampling rate, a resampling step is performed. Kachuee et al. [22] applied a filtration methodology on both ECG and PPG signals. The signals are decomposed using DWT with a db8 to level 10 decomposition. Thenoisycomponents are zeroed and the remaining coefficients are the details of the signal, and these coefficients are obtained based on the soft Rigrsurethresholding methodology [33]. Moreover, Wang et al. [29] designed a filtration method for both signals to remove the main noise components. The ECG and PPG signals are analyzed using FFT to determine the concentration of the energy and noise components. Finally, a Kaiser window-based FIR filter was applied as it has desirable features in both time and frequency domains.

Another pre-processing methodology was applied by Tanveer and Hasan [24] is based on TQWT to remove the baseline wandering [34, 35]. Liu et al. [25] applied a filtration method based on the Butterworth band-pass on each of the ECG and PPG signals. The method ends with adaptive thresholding. Moreover, Xu et al. [26] applied a preprocessing method based on one bandpass filter of FIR and IIR filter. Yao et al. [27] depended on wavelet decomposition using debauchee ' $\mathrm{db} 5$ ' with level 8 decomposition. The decomposition coefficients were filtered using a low pass filter before the reconstruction. Finally, Vandecasteele et al. [30] applied a specific pre-processing methodology for each signal separately. The powerline interference in the ECG signals is removed by applying a notch-filter [36], whereas in the PPG signals a linear-phase finite impulse response is applied followed by a low-pass differentiator filter to remove the sudden upslope in the PPG pulses.

\subsection{Feature extraction}

This phase determines the main features that must be obtained from the ECG and PPG signals. The features obtained from the signals depend on the purpose or the type of disease or diagnosis that is going to be estimated. In other words, in hypertension and the cuff blood pressure studies, the feature extraction phase depends on a set of indicators such as the PTT or sometimes known as PAT, PIR, and a set of features from ECG and PPG signals. Moreover, for the detection of the epileptic seizure, respiratory sinus arrhythmia, and even in the identification of the alcohol consumption there exist other types of indicators such as the HR, HRV, sometimes called RR interval (RRI) [37], and PRV or sometimes called PP interval (PPI).

In the hypertension studies, the PAT feature was extracted with other morphology PPG features [18]. The morphological features are about 135 features obtained from PPG, video plethysmography (VPG), and acceleration plethysmography (APG) signals. Lan et al. [19] for the prediction of hypertension used features based on HRV. In the estimation of the cuffless BP, the PTT and PIR measurements were the most commonly used indicators. Sharifi et al. [23] that was proposed for the estimation of the cuffless BP proposed a new indicator known as pulse wave velocity (PWV). It is estimated that SBP is considered as the summation of the DBP and pulse pressure (PP) which holds high-frequency parts. The SBP can have different formulas for calculation. Moreover, MBP is calculated from the terms of SBP and DBP. Other measurements were also proposed based on PTT and PIR for the BP estimation. Studies Ding et al. [21] and Kachuee et al. [22] estimated DBP and SBP in terms of the PTT and PIR.

A deep learning approach was based on the artificial neural network- long short-term memory (ANN-LSTM) [38] network for extracting features from a successive ECG and PPG signal form for the estimation of the BP. The network holds two layers of LSTM and 1 ANN layer. Moreover, in the estimation of the BP, Liu et al. [25] defined15 features based on the time delay from the R peak of the ECG to the PPG, foot of PPG, and the first derivative of the PPG [39]. Xu et al. [26] obtained features for the estimation of BP. These features are the amplitude of the systolic, diastolic peaks, the time interval 
between two neighboring systolic and diastolic peaks, and others based on these peaks. In the BP estimation, Yao et al. [27] extracted 70 features, and based on a mutual information coefficient analysis an optimized subset is extracted, and this optimized subset showed the most discriminant features for estimation.

The main features extracted for the identification of alcohol consumption are classified as ECG features and PPG features. In the estimation or the evaluation of the coherence between the ECG and PPG parameters, Wang et al. [29] selected several types of features based on the HRV and PRV. The RRI and the PPI were obtained from the ECG and PPG signals [40]. Then, two more features were extracted based on the RRI and the PPI such as the SDNN, and the standard deviation of all peak-to-peak intervals (SDPP).

In the prediction of the epileptic seizure, Vandecasteele et al. [30] used the HRV and PRV as inputs. Three main features were obtained based on the HR [41]. In the identification of alcohol consumption, the HRV and PRV were applied, but they were defined in different terms known as RRI and PTT respectively. Finally, some studies were applied for the cuffless BP estimation presented a calibration methodology. This methodology is used to eliminate the correlation between the subject's BP and PTT. It is reliable for short intervals of time and can be applied for monitoring of the BP as exercise tests $[42,43]$.

\subsection{Classification}

This phase is the final process for the identification, estimation, or prediction of the features obtained from ECG and PPG signals. Some studies applied some classifiers for deducing the final accuracy, while others used regression techniques to estimate the error performance. On one hand, the main common classifiers used in the studies that depend on the ECG and PPG signals are the AdaBoost tree (ADT), BT, KNN, SVM [44], RF, and multiple instances learning algorithms (MIL), BNN. On the other hand, the main common regression methods are the RT, decision tree (DT), support vector regression (SVR), ridge regression, MARS, random forest regression (RFR), linear regression (LR), and the regularized linear regression (RLR) [45]. MARS showed an efficient performance as it is preserved to be a nonlinear regression model. It takes the merits of the prediction and modeling methods. It is a powerful regression method and it can illustrate efficiently the hidden and nonlinear pattern in the data as it is considered to be a generalized form of stepwise linear regression.

\subsection{Performance measurements}

Several statistical measurements were used in the studies with the aim is to evaluate the performance. The measurements used are the true positive (TP), false positive (FP), true negative (TN), false negative (FN), sensitivity, false positive, positive predictive value (PPV), receiver operating characteristic (ROC), F1score, recall, precision, and accuracy. These measurements are the most statistical parameters used in the prediction and classification problem. Some studies applied other types of performance measurements such as the MAD, RMSE, correlation coefficient (CC), mean (M), standard deviation (std), and PRD.

\section{DISCUSSION AND LIMITATIONS}

This study presents the main applications that use the combination of the ECG and PPG signal. Each application depends on a set of phases such as capturing data, filtering the signal, extracting features, and classification or estimation. In the data acquisition phase, there existed several types of devices that can capture the ECG and PPG signals accurately. It can be seen that the main databases used in the applications based on the combination of the ECG and PPG signals are the MIMIC I and II. In the pre-processing phase, the DWT, VMD, and TQWT were the most common filtering methods that produced both signals in a clean shape and showed the highest signal-to-noise ratio (SNR). The former pre-processing methods can remove the three main noises that occur in both signals which are the baseline drift, powerline interference, and high-frequency noise in an efficient performance. Moreover, in the feature extraction phase, the HRV, PRV, PTT, and PIR were the main extracted features. The combination between the former features is applied in most of the studies, and even the mean, the standard deviation of these features are obtained in some studies to gain robust features. In the classification phase, SVM, ANN, and BNN classifiers showed the highest performance in the classification, while in the regression methods, MARS and AdaBoost had the lowest error performance. The main applications that existed based on the combination of the ECG and PPG signals are hypertension, alcohol consumption, and estimation of the respiratory sinus arrhythmia, cuffless BP, and epilepsy seizure detection. Table 1 shows the main studies that combined ECG and PPG signals for different applications. Each column in the table represents the author, year, data acquisition, filtration, features, classification or regression, accuracy performance, and the application.

It can be seen that a few applications applied both signals. This can lead to some limitations and open issues. These limitations can be illustrated in four main points. The first limitation is the applications and purposes that used both signals combined are hypertension, alcohol consumption, and estimation of the respiratory sinus arrhythmia, cuffless BP, and epilepsy seizure detection. These are considered to be a limited number of applications. Several types of diseases can be detected using both ECG and PPG signals. For instance, diseases such as asystole, extreme bradycardia or tachycardia, ventricular tachycardia, and ventricular flutter or fibrillation can be predicted with high accuracy using both signals. Without a doubt, it can be verified that the accuracy performance in diagnosing a disease based on combined physiological signals will achieve higher accuracy than the diagnosis based on a single signal. The features obtained from multiple signals will contribute to providing more information about the disease that will be estimated or predicted. The second limitation is that there are a few deep learning approaches that were applied in the studies that are based on the combination of ECG and PPG signals. In other words, deep learning can learn and train from data easily without any handcrafted features, and if it is applied to the combination of ECG and PPG signals, the performance of the diagnosis, estimation, even prediction will be improved.

The third limitation is that the number of classifiers or regression models that are applied in the studies that use both signals is limited. There exist various types of recent machine learning classifiers such as sparse representation classifiers (SRC), multi adaptive sparse representation classifiers, XGBoost classifiers [46], and many others. Moreover, there 
exist different regression models rather than the linear and the logistic and these regression models are polynomial, quantile, and lasso models. Finally, the last limitation is that most of the studies used HRV, PRV, PTT, PIR, and a set of features formed from the combination of ECG and PPG peaks and intervals.

Table 1. Summary of different related studies that combines ECG and PPG signals

\begin{tabular}{|c|c|c|c|c|c|c|}
\hline $\begin{array}{c}\text { Authors \& } \\
\text { Year }\end{array}$ & Dataset & Pre-processing & Feature Extraction & $\begin{array}{c}\text { Classification/ } \\
\text { Regression } \\
\end{array}$ & Results & Application \\
\hline $\begin{array}{l}\text { Liang et al. } \\
\qquad[18]\end{array}$ & $\begin{array}{l}\text { MIMIC } \\
\text { Database } \\
121 \\
\text { Subjects }\end{array}$ & $\begin{array}{l}\text { Excluding the } \\
\text { missing peaks, } \\
\text { pulsus bisferiens }\end{array}$ & $\begin{array}{c}\text { PAT Features } \\
\text { PPG Features } \\
\text { The mix between PAT and PPG } \\
\text { features }\end{array}$ & $\begin{array}{l}\text { ABT, BT, LR, } \\
\text { KNN }\end{array}$ & $\begin{array}{c}\mathrm{N} \text { vs PTKNN }=84.34 \% \\
\mathrm{~N} \text { vs HTKNN }=94.84 \% \\
\mathrm{~N} \text { vs PT vs HTKNN } 88.49 \%\end{array}$ & $\begin{array}{l}\text { Hypertension } \\
\text { Prediction }\end{array}$ \\
\hline Lan et al. [19] & $\begin{array}{l}43 \text { Subjects } \\
30 \text { Train, } \\
13 \text { Test }\end{array}$ & $\begin{array}{l}\text { A pre-filter } \\
\text { mechanism }\end{array}$ & $\begin{array}{l}\text { SDNN, RMSSD, nLF } \\
\text { nHF, MeanRR, LFHF }\end{array}$ & MILL & $\begin{array}{c}\mathrm{SDNN}=85.47 \%, \mathrm{RMSSD}= \\
35.90 \%, \mathrm{MeanRR}=65.81 \% \\
\mathrm{LFHF}=70.94 \%, \mathrm{nLF}= \\
51.71 \%, \mathrm{nHF}=64.96 \%\end{array}$ & $\begin{array}{l}\text { Hypertension } \\
\text { Prediction }\end{array}$ \\
\hline $\begin{array}{l}\text { Ghosh et al. } \\
\text { [20] }\end{array}$ & $\begin{array}{l}14 \text { Subjects } \\
\text { Motion and } \\
\text { without } \\
\text { motion }\end{array}$ & $\begin{array}{l}\text { Pre-processed } \\
\text { ECG and PPG } \\
\quad \text { signal }\end{array}$ & $\begin{array}{l}\text { PTT features + calibration + } \\
\text { with sparsity and without } \\
\text { sparsity to obtain BP values }\end{array}$ & $\begin{array}{l}\text { RMSE on the } \\
\text { SBP and DBP }\end{array}$ & $\begin{array}{l}\text { Mean and Standard deviation } \\
\text { Seated: } 0.07 \pm 5.8 \\
\text { mmHgWalking: } 4.4 \pm \\
\text { 20.9Cycling: } 10.2 \pm 16.0\end{array}$ & Cuffless BP \\
\hline $\begin{array}{l}\text { Ding et al. } \\
\quad[21]\end{array}$ & 27 Subjects & $\begin{array}{l}\text { Pre-processed } \\
\text { ECG and PPG } \\
\quad \text { signal }\end{array}$ & PTT and PIR feature extraction & - & $\begin{array}{c}\text { DBPMAD: } 4.09 \\
\text { mmHg,MBPMAD: } 3.18 \\
\text { mmHg } \\
\text { SPBMAD: } 3.18 \mathrm{mmHg} \\
\text { First Type (SBP)AdaBoost: }\end{array}$ & Cuffless BP \\
\hline $\begin{array}{l}\text { Kachuee et al. } \\
\text { [22] }\end{array}$ & $\begin{array}{l}\text { MIMIC-II } \\
\text { Version } 3 \\
\text { dataset } \\
3662 \\
\text { Records }\end{array}$ & $\begin{array}{l}\text { DWT at } \mathrm{N}=10 \\
\text { with db8 }+ \text { Soft } \\
\text { threshold } \\
\text { Zeroing } 0 \sim 0.25 \\
\text { Hz, Zeroing } \\
250 \sim 500 \mathrm{~Hz}\end{array}$ & $\begin{array}{c}\text { First Type: (HR, Augmentation } \\
\text { index, Arterial Stiffness index, } \\
\text { PAT, Inflection point Area } \\
\text { ratio) } \\
\text { Second Type:Features extracted } \\
\text { from ECG and PPG in Time } \\
\text { domain }\end{array}$ & $\begin{array}{l}\text { RLF, DT, SVM } \\
\text { AdaBoostRFR }\end{array}$ & $\begin{array}{c}\text { 11.17, RF: } 9.87 \\
\text { Second Type (SBP)AdaBoost: } \\
\text { 11.87,RF: } 10.09 \\
\text { First Type (DBP)AdaBoost: } \\
\text { 5.35, RF: } 5.71 \\
\text { Second Type } \\
\text { (DBP)AdaBoost: } 5.78, \text { RF: } \\
6.06\end{array}$ & Cuffless BP \\
\hline $\begin{array}{l}\text { Sharifi et al. } \\
\text { [23] }\end{array}$ & $\begin{array}{l}\text { MIMIC-II } \\
\text { version } 3 \\
\text { dataset } \\
3663 \\
\text { Records }\end{array}$ & VMD & PTT and PIR feature extraction & MARS & $\begin{array}{c}\text { DBP MAD: } 4.86 \mathrm{mmHg} \\
\text { PRD: } 7.23 \mathrm{mmHg} \\
\text { MBP MAD: } 3.63 \mathrm{mmHg} \\
\text { PRD: } 9.44 \mathrm{mmHg} \\
\text { SBP MAD: } 7.83 \mathrm{mmHg} \\
\text { PRD: } 9.82 \mathrm{mmHg}\end{array}$ & Cuffless BP \\
\hline $\begin{array}{l}\text { Tanveer and } \\
\text { Hasan [24] }\end{array}$ & $\begin{array}{l}\text { MIMIC- I } \\
39 \text { Subjects }\end{array}$ & T-QWT & $\begin{array}{l}\text { ECG and PPG Waveform } \\
\text { fragments + LSTM }\end{array}$ & ANN & $\begin{array}{c}\text { SBP MAE: } 1.10 \mathrm{mmHg} \text {, } \\
\text { RMSE: } 1.56 \mathrm{mmHg} \\
\text { DBP MAE: } 0.58 \mathrm{mmHg} \\
\text { RMSE: } 0.85 \mathrm{mmHg}\end{array}$ & Cuffless BP \\
\hline Liu et al. [25] & 35 Subjects & $\begin{array}{l}\text { Butterworth band- } \\
\text { pass filtering }\end{array}$ & $\begin{array}{l}15 \text { features were selected from } \\
\text { ECG and PPG }\end{array}$ & $\begin{array}{c}\text { DT, SVR, } \\
\text { Adaboost, RF }\end{array}$ & $\begin{array}{l}\text { Mean and Standard deviation } \\
\text { RF has the lowest mean } \\
\text { square error } \\
\text { SBP RF: } 5.87 \pm 3.13 \text { and } \\
3.52 \pm 1.38 \mathrm{mmHg}\end{array}$ & Cuffless BP \\
\hline $\mathrm{Xu}$ et al. [26] & $\begin{array}{c}7 \\
\text { volunteers } \\
4 \text { male, } 3 \\
\text { females }\end{array}$ & $\begin{array}{l}\text { One bandpass } \\
\text { filter composed of } \\
\text { HPF and LPF }\end{array}$ & $\begin{array}{l}\text { Detect R peak, compute PTT, } \\
\text { find systolic and diastolic peaks, } \\
\text { Derivation of both peaks to find } \\
\text { the dicrotic notch }\end{array}$ & $\mathrm{BNN}$ & $\begin{array}{c}\text { Mean and Standard deviation } \\
\text { SBP }-0.41 \pm 2.02 \mathrm{mmHg} \\
\text { DBP } 0.46 \pm \mathrm{mmHg}\end{array}$ & Cuffless BP \\
\hline $\begin{array}{l}\text { Yao et al. } \\
\text { [27] }\end{array}$ & $\begin{array}{l}\text { MIMIC-II } \\
2000 \\
\text { records } \\
500 \\
\text { samples } \\
\text { from each } \\
\text { record }\end{array}$ & $\begin{array}{c}\text { Wavelet } \\
\text { decomposition } \\
\text { with db5 level } 8 \\
+ \text { low pass filter }\end{array}$ & $\begin{array}{l}70 \text { features were extracted from } \\
\text { the ECG and PPG signals, an } \\
\text { optimized selection feature set } \\
\text { was extracted from the } 70 \\
\text { features }\end{array}$ & $\begin{array}{l}\text { SVM, } \\
\text { AdaBoost } \\
\text { LR, DT } \\
\text { RT, Ridge } \\
\text { regression }\end{array}$ & $\begin{array}{l}\text { AdaBoost showed the highest } \\
\text { performance } \\
\text { SBP MAE: } 8.02 \text { RMSE: } \\
10.73 \text { r: } 0.80 \\
\text { MBP MAE: } 5.09 \text { RMSE: } \\
\begin{array}{ll}7.52 & \text { r: } 0.69 \\
\text { DBP } & \text { MAE: } 4.54 \text { RMSE: } \\
6.88 & \text { r: } 0.71\end{array}\end{array}$ & Cuffless BP \\
\hline Jan et al. [28] & 30 Subjects & $\begin{array}{l}\text { Pre-processed } \\
\text { ECG and PPG } \\
\quad \text { signal }\end{array}$ & Parameters from HRV and PRV & $\begin{array}{l}\text { SDNN, SDPP, } \\
\text { Ratio } \\
\text { HRV of } \\
\text { PRV }\end{array}$ & $\begin{array}{l}\text { The ratio of HRV was higher } \\
\text { than PVR in all of the classes } \\
\text { and even during the rest }\end{array}$ & $\begin{array}{c}\text { RSA } \\
\text { estimation }\end{array}$ \\
\hline $\begin{array}{l}\text { Wang et al. } \\
\text { [29] }\end{array}$ & 10 Subjects & $\begin{array}{l}\text { FFT + Kaiser } \\
\text { window-based } \\
\text { FIR filter }\end{array}$ & $\begin{array}{c}\text { Featuresfrom ECG, PPG, and } \\
\text { Combination of features from } \\
\text { them }\end{array}$ & SVM & Accuracy $=95 \%$ & $\begin{array}{l}\text { Alcohol } \\
\text { consumption }\end{array}$ \\
\hline $\begin{array}{l}\text { Vandecasteele } \\
\text { et al. [30] }\end{array}$ & 11 Subjects & $\begin{array}{l}\text { ECG: Notch filter } \\
\text { PPG: Linear } \\
\text { Low pass } \\
\text { filter }\end{array}$ & $\begin{array}{c}\text { PRV }+ \text { HRV } \\
+ \text { Epileptic Seizure detection } \\
\text { algorithm }\end{array}$ & - & $\begin{array}{c}\text { Sensitivity Wearable PCG = } \\
70 \% \\
\text { Wearable ECG }=32 \% \\
\text { Hospital ECG }=57 \%\end{array}$ & $\begin{array}{l}\text { Epileptic } \\
\text { Seizure } \\
\text { Prediction }\end{array}$ \\
\hline
\end{tabular}

\section{CONCLUSIONS AND FUTURE WORK}

Several researchers around the world address different problems that are related to the ECG and PPG signals combined. Therefore, they require more information in the analysis of the signals at different stages. Even though there exist different studies that applied both signals in different applications and problems, but there are still lots of applications that both signals can be applied in. In this paper, a comprehensive study has been provided, and the datasets, 
preprocessing, feature extraction, classification, and applications have been handled using different aspects. A few numbers of recommendations are concluded. It is recommended that the PPG fragment or the ECG fragment as a whole be used in the phase of feature extraction. The main reasons for this are that the PPG and the ECG fragments hold a lot of samples and features that can benefit the type of application the signals are conducted in, and in addition to this, every disease or arrhythmia that affect the heart will have a different shape in the ECG and PPG fragment. The fragment or the frequency of the fragment in both signals can have a huge influence on the prediction and estimation performance. It is also recommended from the research community that the heart datasets available online should include the ECG, PPG, phonocardiogram (PCG), and any other signals related to the heart for a single individual because a few datasets are available online have multiple signals obtained from the heart In other words, even if the individual is a patient or in a good health situation, the combination between his signals and the variances obtained from the signals can enhance the application's performance. This can also provide a better estimate in different applications because each individual has multiple signals related to the heart. In this way, the interested researchers can have the recent developments and applications on various aspects of ECG and PPG signals combined from a single source.

\section{REFERENCES}

[1] Kannampallil, T.G., Schauer, G.F., Cohen, T., Patel, V.L. (2011). Considering complexity in healthcare systems. Journal of Biomedical Informatics, 44(6): 943-947. https://doi.org/10.1016/j.jbi.2011.06.006

[2] Wendt, C., Frisina, L., Rothgang, H. (2009). Healthcare system types: A conceptual framework for comparison. Social Policy \& Administration, 43(1): 70-90. https://doi.org/10.1111/j.1467-9515.2008.00647.x

[3] Manikandan, M.S., Soman, K.P. (2012). A novel method for detecting R-peaks in electrocardiogram (ECG) signal. Biomedical Signal Processing and Control, 7(2): 118128. https://doi.org/10.1016/j.bspc.2011.03.004

[4] Saritha, C., Sukanya, V., Murthy, Y.N. (2008). ECG signal analysis using wavelet transforms. Bulgarian Journal of Physics, 35(1): 68-77.

[5] Gil, E., Bailón, R., Vergara, J.M., Laguna, P. (2010). PTT variability for discrimination of sleep apnea related decreases in the amplitude fluctuations of PPG signal in children. IEEE Transactions on Biomedical Engineering, 57(5): https://doi.org/10.1109/TBME.2009.2037734

[6] Schäfer, A., Vagedes, J. (2013). How accurate is pulse rate variability as an estimate of heart rate variability? A review on studies comparing photoplethysmographic technology with an electrocardiogram. International Journal of Cardiology, 166(1): 15-29. https://doi.org/10.1016/j.ijcard.2012.03.119

[7] Bent, B., Goldstein, B.A., Kibbe, W.A., Dunn, J.P. (2020). Investigating sources of inaccuracy in wearable optical heart rate sensors. NPJ Digital Medicine, 3(1): 19. https://doi.org/10.1038/s41746-020-0226-6

[8] Pielmuş, A.G., Osterland, D., Klum, M., Tigges, T., Feldheiser, A., Hunsicker, O., Orglmeister, R. (2017). Correlation of arterial blood pressure to synchronous piezo, impedance and photoplethysmographic signal features: Investigating pulse wave features and transit times. Current Directions in Biomedical Engineering, 3(2): 749-753. https://doi.org/10.1515/cdbme-20170158

[9] Bruno, R.M., Duranti, E., Ippolito, C., et al. (2017). Different impact of essential hypertension on structural and functional age-related vascular changes. Hypertension, $69(1)$ : 71-78. https://doi.org/10.1161/HYPERTENSIONAHA.116.08 041

[10] Mukkamala, R., Hahn, J.O., Inan, O.T., Mestha, L.K., Kim, C.S., Töreyin, H., Kyal, S. (2015). Toward ubiquitous blood pressure monitoring via pulse transit time: theory and practice. IEEE Transactions on Biomedical Engineering, 62(8): 1879-1901. https://doi.org/10.1109/TBME.2015.2441951

[11] Forsgren, L., Beghi, E., Oun, A., Sillanpää, M. (2005). The epidemiology of epilepsy in Europe-a systematic review. European Journal of Neurology, 12(4): 245-253. https://doi.org/10.1111/j.1468-1331.2004.00992.x

[12] Fisher, R.S., Blum, D.E., DiVentura, B., Vannest, J., Hixson, J.D., Moss, R., French, J.A. (2012). Seizure diaries for clinical research and practice: limitations and future prospects. Epilepsy \& Behavior, 24(3): 304-310. https://doi.org/10.1016/j.yebeh.2012.04.128

[13] Massé, F., Bussel, M. V., Serteyn, A., Arends, J., Penders, J. (2013). Miniaturized wireless ECG monitor for realtime detection of epileptic seizures. ACM Transactions on Embedded Computing Systems (TECS), 12(4): 1-21. https://doi.org/10.1145/2485984.2485990

[14] Ryan, J.M., Howes, L.G. (2002). Relations between alcohol consumption, heart rate, and heart rate variability in men. Heart, 88(6): 641-642. https://doi.org/10.1136/heart.88.6.641

[15] Lal, S.K., Mihailidou, A.S., Cejnar, M., Henderson, R.J., Jones, M., Hunyor, S.N. (1993). Continuous, noninvasive volume-clamp blood pressure: Determinants of performance. Journal of Hypertension, 11(12): 14131422. https://doi.org/10.1097/00004872-19931200000014

[16] Senturk, U., Polat, K., Yucedag, I. (2020). A noninvasive continuous cuffless blood pressure estimation using dynamic recurrent neural networks. Applied Acoustics, 170: 107534. https://doi.org/10.1016/j.apacoust.2020.107534

[17] Kox, M., Pompe, J.C., van der Hoeven, J.G., Hoedemaekers, C.W., Pickkers, P. (2011). Influence of different breathing patterns on heart rate variability indices and reproducibility during experimental endotoxaemia in human subjects. Clinical Science, 121(5): 215-222. https://doi.org/10.1042/CS20110027

[18] Liang, Y., Chen, Z., Ward, R., Elgendi, M. (2018). Hypertension assessment via ECG and PPG signals: An evaluation using MIMIC database. Diagnostics, 8(3): 65. https://doi.org/10.3390/diagnostics8030065

[19] Lan, K.C., Raknim, P., Kao, W.F., Huang, J.H. (2018). Toward hypertension prediction based on PPG-derived HRV signals: A feasibility study. Journal of Medical Systems, 42(6): 1-7. https://doi.org/10.1007/s10916018-0942-5

[20] Ghosh, S., Banerjee, A., Ray, N., Wood, P.W., Boulanger, P. Padwal, R. (2016). Continuous blood pressure prediction from pulse transit time using ECG 
and PPG signals. In 2016 IEEE Healthcare Innovation Point-Of-Care Technologies Conference (HI-POCT), pp. 188-191. https://doi.org/10.1109/HIC.2016.7797728

[21] Ding, X.R., Zhang, Y.T., Liu, J., Dai, W.X., Tsang, H.K. (2015). Continuous cuffless blood pressure estimation using pulse transit time and photoplethysmogram intensity ratio. IEEE Transactions on Biomedical Engineering, 63(5): https://doi.org/10.1109/TBME.2015.2480679

[22] Kachuee, M., Kiani, M.M., Mohammadzade, H., Shabany, M. (2016). Cuffless blood pressure estimation algorithms for continuous healthcare monitoring. IEEE Transactions on Biomedical Engineering, 64(4): 859-869. https://doi.org/10.1109/TBME.2016.2580904

[23] Sharifi, I., Goudarzi, S., Khodabakhshi, M.B. (2019). A novel dynamical approach in continuous cuffless blood pressure estimation based on ECG and PPG signals. Artificial Intelligence in Medicine, 97: 143-151. https://doi.org/10.1016/j.artmed.2018.12.005

[24] Tanveer, M.S., Hasan, M.K. (2019). Cuffless blood pressure estimation from electrocardiogram and photoplethysmogram using waveform-based ANNLSTM network. Biomedical Signal Processing and Control, 51: 382-392. https://doi.org/10.1016/j.bspc.2019.02.028

[25] Liu, Z., Zhou, B., Li, Y., Tang, M., Miao, F. (2020). Continuous blood pressure estimation from electrocardiogram and photoplethysmogram during arrhythmias. Frontiers in Physiology, 11: 1126. https://doi.org/10.3389/fphys.2020.575407

[26] Xu, Z., Liu, J., Chen, X., Wang, Y., Zhao, Z. (2017). Continuous blood pressure estimation based on multiple parameters from electrocardiogram and photoplethysmogram by Back-propagation neural network. Computers in Industry, 89: 50-59. https://doi.org/10.1016/j.compind.2017.04.003

[27] Yao, L.P., Pan, Z.L. (2021). Cuff-less blood pressure estimation from photoplethysmography signal and electrocardiogram. Physical and Engineering Sciences in Medicine, 44(2): 397-408 https://doi.org/10.1007/s13246-021-00989-1

[28] Jan, H.Y., Chen, M.F., Fu, T.C., Lin, W.C., Tsai, C.L., Lin, K.P. (2019). Evaluation of coherence between ECG and PPG-derived parameters on heart rate variability and respiration in healthy volunteers with/without controlled breathing. Journal of Medical and Biological Engineering, 39(5): 783-795 https://doi.org/10.3389/fphys.2020.00867

[29] Wang, W.F., Yang, C.Y.,Wu, Y.F. (2018). SVM-based classification method to identify alcohol consumption using ECG and PPG monitoring. Personal and $\begin{array}{lll}\text { Ubiquitous } & \text { Computing, 22(2): } 275-287 .\end{array}$ https://doi.org/10.1007/s00779-017-1042-0

[30] Vandecasteele, K., De Cooman, T., Gu, Y., Cleeren, E., Claes, K., Paesschen, W.V., Hunyadi, B. (2017). Automated epileptic seizure detection based on wearable ECG and PPG in a hospital environment. Sensors, 17(10): 2338. https://doi.org/10.3390/s17102338

[31] MIMIC

database: https://physionet.org/content/mimicdb/1.0.0/, accessed on 4 April 2021.

[32] Dragomiretskiy, K., Zosso, D. (2013). Variational mode decomposition. IEEE Transactions on Signal Processing,
62(3):

$531-544$

https://doi.org/10.1109/TSP.2013.2288675

[33] Singh, B.N., Tiwari, A.K. (2006). Optimal selection of wavelet basis function applied to ECG signal denoising. Digital Signal Processing, 16(3): 275-287. https://doi.org/10.1016/j.dsp.2005.12.003

[34] Donoho, D.L. (1995). De-noising by soft-thresholding. IEEE Transactions on Information Theory, 41(3): 613627. https://doi.org/10.1109/TSP.2011.2143711

[35] Selesnick, I.W. (2011). Wavelet transforms with tunable Q-factor. IEEE Transactions on Signal Processing, 59(8): 3560-3575. https://doi.org/10.1109/TSP.2011.2143711

[36] Varon, C., Jansen, K., Lagae, L., Van Huffel, S. (2015). Can ECG monitoring identify seizures? Journal of Electrocardiology, 48(6): 1069-1074. https://doi.org/10.1016/j.jelectrocard.2015.08.020

[37] Bassiouni, M., Khaleefa, W., El-Dahshan, E.A., Salem, A.B.M. (2016). A machine learning technique for person identification using ECG signals. Int. J. Appl. Phys, 1: 37-41.

[38] Schuster, M., Paliwal, K.K. (1997). Bidirectional recurrent neural networks. IEEE Transactions on Signal Processing, 45(11): 2673-2681. https://doi.org/10.1109/78.650093

[39] Pandit, J.A., Lores, E., Batlle, D. (2020). Cuffless blood pressure monitoring: promises and challenges. Clinical Journal of the American Society of Nephrology, 15(10): 1531-1538. https://doi.org/10.2215/CJN.03680320

[40] Miao, F., Liu, Z.D., Liu, J.K., Wen, B., He, Q.Y., Li, Y. (2019). Multi-sensor fusion approach for cuff-less blood pressure measurement. IEEE Journal of Biomedical and Health Informatics, 24(1): 79-91. https://doi.org/10.1109/JBHI.2019.2901724

[41] De Cooman, T., Varon, C., Hunyadi, B., Van Paesschen, W., Lagae, L., Van Huffel, S. (2017). Online automated seizure detection in temporal lobe epilepsy patients using single-lead ECG. International Journal of Neural Systems, 27(07): 1750022. https://doi.org/10.1142/S0129065717500228

[42] Gesche, H., Grosskurth, D., Küchler, G., Patzak, A. (2012). Continuous blood pressure measurement by using the pulse transit time: Comparison to a cuff-based method. European Journal of Applied Physiology, 112(1) 309-315. https://doi.org/10.1007/s00421-011-1983-3

[43] Revett, K., Gorunescu, F., Salem, A.B., El-Dahshan, E.S. (2009). Evaluation of the feature space of an erythematosquamous dataset using rough sets. Annals of the University of Craiova-Mathematics and Computer Science Series, 36(2): 123-130.

[44] Mohsen, H., El-Dahshan, E., El-Horbaty, E., Salem, A. (2017). Brain tumor type classification based on support vector machine in magnetic resonance images. Annals of "Dunarea De Jos" University Of Galati, Mathematics, Physics, Theoretical mechanics, Fascicle II, Year IX (XL), (1).

[45] Hastie, T., Tibshirani, R., Friedman, J. (2009). The elements of statistical learning: Data mining, inference, and prediction. Springer Science \& Business Media.

[46] El-Bakry, S.Y., El-Dahshan, E.S., El-Bakry, M.Y. (2011). Total cross section prediction of the collisions of positrons and electrons with alkali atoms using Gradient Tree Boosting. Indian Journal of Physics, 85(9): 14051415. https://doi.org/10.1007/s12648-011-0162-z 